\title{
Large non-adiabatic hole polarons and matrix element effects in the angle-resolved photoemission spectroscopy of dielectric cuprates
}

\author{
A.S. Moskvin, E.N. Kondrashov, V.I. Cherepanov
}

Department of Theoretical Physics, Ural State University 620083, Ekaterinburg, Russia

\begin{abstract}
It has been made an extention of the conventional theory based on the assumption of the well isolated Zhang'Rice singlet to be a first electron-removal state in dielectric copper oxide. One assumes the photohole has been localised on either small (pseudo)Jahn-Teller polaron or large non-adiabatic polaron enclosed one or four to five $\mathrm{CuO}_{4}$ centers, respectively, with active one-center valent $\left({ }^{1} A_{1 g}-{ }^{1,3} E_{u}\right)$ manifold. In the framework of the cluster model we have performed a model microscopic calculation of the $\mathbf{k}$-dependence of the matrix element effects and 'photon polarization effects for the angle-resolved photoemission in dielectric cuprate like $\mathrm{Sr}_{2} \mathrm{CuO}_{2} \mathrm{Cl}_{2}$. We show that effects like the "remnant Fermi surface" detected in ARPES experiment for $\mathrm{Ca}_{2} \mathrm{CuO}_{2} \mathrm{Cl}_{2}$ may be, in fact, a reflection of the matrix element effects, not a reflection of the original band-structure Fermi surface, or the strong antiferromagnetic correlations. The measured dispersion-like features in the low-energy part of the ARPES spectra may be a manifestation of the complex momentum-dependent spectral line-shape of the large PJT polaron response, not the dispersion of the well-isolated Zhang-Rice singlet in antiferromagnetic matrix.
\end{abstract}

\section{Introduction}

Angle-resolved photoemission spectroscopy (ARPES) is considered to be a key experiment to elucidate a number of principal issues of electronic theory related to the unconventional properties of cuprates, manganites, nickellates, bismuthates, and other strongly correlated oxides. These are the quasiparticle dispersion, the Fermi surface, ', (pseudo)gap behaviour and other ones. Many researchers consider namely ARPES data to be a main evidence in favour of either model of electronic structure and energy spectrum.

However, the current practice of ARPES activity in oxides does not justify these expectations. Up to now the ARPES measurements are treated as a rule in the framework of oversimplified "three-stage" approach. The first '" experimental" one relates straightforwardly to measurements of the $(E, \mathbf{k})$-dependencies of photocurrent intensity. Any problems here are related to a sample preparation, experimental setup, and attempts to avoid such external "parasitic phenomena as a charging effect. The very "dangerous" second "experimental-theoretical" stage implies the preliminary treatment and often incorporates a "hidden" interpretation based on a number of seemingly inviolable 'statements like:

1. ARPES data in a wide spectral range could be self-consistently described by the conventional band models. 'Electronic structure and energy spectrum near the Fermi level are formed by Landau quasiparticles with Fermi 'statistics which fill an energy band.

2. The peaks position for the photocurrent intensity links to the peaks in the quasiparticle spectral density. In other words, ARPES essentially measures the one-particle spectral function of the initial state.

3. Some authors go further and make use of the standard method which provides the information on the Fermi surface from ARPES data in the conventional band metals. First, one find the occupation probability, $n(\mathbf{k})$, by 'integrating the ARPES spectral function $A(\mathbf{k}, E)$ over energy. Experimentally, one choses an energy window for integration, thus becoming the relative $n(\mathbf{k})$. Then, the drop of the relative $n(\mathbf{k})$ is used to determine the Fermi 'surface. In practice, one defines $k_{F}$ as the locus of points of maximum gradient of $n_{\vec{k}}$ or as the point of steepest descent in the relative $n(\mathbf{k})$. Naturally, this method implies the simple metallic-like electronic structure, where the 'identification of a Fermi surface is convincing.

In our opinion, this "band-like" paradigm supported only by the simplest model like that of free electrons, turns 'out to be at least questionable in all points in the case of strongly correlated oxides. Firstly, an ability of band models, even modified like LDA+U [1], to yield a relevant description of electron spectra for strongly correlated oxides is merely a misleading, at present there are not convincing examples for such a description, in particular, for the lowest-lying occupied band, or the so-called first electron-removal states. The typical errors in locating the final states for ab initio band structures are generally expected to be of the order of a few eV's (!). Large number of experimental data evidences in favour of unconventional "non-Landau " nature of quasiparticles and occurrence of unusual correlations in cuprates and other oxides. Traditional interpretation of ARPES data like a simple accordance between the photointensity peak position and the quasiparticle spectral density peak implies a full neglect of several factors each of which is capable to result in crucial reconsideration of the ARPES data: i) the matrix element effects, or the intensity dispersion; ii) surface effects; iii) effects of multiple scattering; iv) effects of finite lifetimes of the initial and final states; v) effects of coupling with phonons, spin excitations and other possible degrees of freedom; vi) effects of configurational interaction; vii) effects of electron inhomogeneity, for instance, in doped cuprates. For insulating samples it is difficult to escape the charging effects.

Importance of matrix element effects and the ARPES intensity dispersion for different cuprates was underlined earlier [2], and a role played by configurational interaction was partially illustrated in Ref. [8] for $\mathrm{Sr}_{2} \mathrm{CuO}_{2} \mathrm{Cl}_{2}$. 
The hole polaron formation due to electron-phonon coupling and its manifestation in ARPES was demonstrated by Alexandrov and Dent [2] for cuprates like Y123 and Y124. One should be noted that all the above mentioned citations relate to the nontraditional approach in ARPES.

The third, purely theoretical stage implies a qualitative and quantitative description of the dispersion law and other quasiparticle properties obtained on the foregoing stage and to be considered as "experimental data" (?!). Unfortunately, too often this stage looks like a formal fitting with either model theory, and with no critical inspection of experimental data. Here one might mention, for instance, numerous papers with theoretical treatment of "experimental" ARPES data for $\mathrm{Sr}_{2} \mathrm{CuO}_{2} \mathrm{Cl}_{2}$ in the framework of various extended $t-J, t-t^{\prime}-J, t-t^{\prime}-t^{\prime \prime}-J$ models [9.10].

Summarizing, we see this "three-stage" approach could result in a natural doubts as to conclusions based on such an oversimplified interpretation.

As a convenient demonstrative model system for ARPES in the insulating layered copper oxides one might be chosen oxychlorides $\mathrm{Sr}_{2} \mathrm{CuO}_{2} \mathrm{Cl}_{2}$ and $\mathrm{Ca}_{2} \mathrm{CuO}_{2} \mathrm{Cl}_{2}$, which are isostructural to famous 214 system $\mathrm{La}_{2} \mathrm{CuO}_{4}$. The oxychloride $\mathrm{Sr}_{2} \mathrm{CuO}_{2} \mathrm{Cl}_{2}$ is one of the most popular model system for insulating phase of the high- $T_{c}$ cuprates and is intensively studied both experimentally and theoretically.

In this tetragonal antiferromagnetic with nearly ideal $\mathrm{CuO}_{2}$ planes there are chlorine atoms instead of apex oxygens with considerably larger $\mathrm{Cu}-\mathrm{Cl}_{\text {apex }}$ separation $(2.86 \AA)$ than that of $\mathrm{Cu}-\mathrm{O}_{\text {apex }}(2.42 \AA)$ in $\mathrm{La}_{2} \mathrm{CuO}$. Hence, in $\mathrm{Sr}_{2} \mathrm{CuO}_{2} \mathrm{Cl}_{2}$ one appears a real opportunity to examine the $\mathrm{CuO}_{2}$ plane states, both copper and oxygen, without "parasitic" contribution of apex oxygens. At present there are a rather large number of experimental data for $\mathrm{Sr}_{2} \mathrm{CuO}_{2} \mathrm{Cl}_{2}$ obtained with the help of optical spectroscopy 11, X-ray photoemission (XPS) 12], ultraviolet photoemission (UPS) [13], X-ray absorption spectroscopy (XAS) 14, 15, electron energy loss spectroscopy (EELS) 16 18], angle-resolved photoemission spectroscopy [5, 6, 9, 21,22]. Similar experimental ARPES results were obtained recently by F. Ronning et al. [20] for $\mathrm{Ca}_{2} \mathrm{CuO}_{2} \mathrm{Cl}_{2}$ which is a full analog of $\mathrm{Sr}_{2} \mathrm{CuO}_{2} \mathrm{Cl}_{2}$.

The authors [9] state: "This is a measurement of the dispersion of a single hole in an antiferromagnetic background, a problem that has been heavily investigated theoretically". It seems, this is a very strong and unambiguous statement, which oversimplifies the problem and does not account of many alternative scenarios. Moreover, the experimental data [9] could hardly considered to be a reliable basis set for any decisive conclusions. Indeed, the spectral dependence of the photocurrent intensity shows a complex nature of the low-energy photo-hole states with a strong and dispersive contribution of the high-lying states.

It is very important to emphasize that, while the ARPES spectra in various oxides displayed as the photointensity against $E$, $\mathbf{k}$ curves show the dispersion-like features (see, e.g. Fig.1, Ref. [9], or Fig.3, Ref. 20]), the "dispersing" states which "peak positions" are plotted are extremely broad, with width comparable to binding energy, and these simply cannot be thought of as quasiparticles. This general point is true at all k's. In addition, in the most part of the Brillouin zone there are no well-defined ARPES peaks at all. Moreover, the observed peak position may rather strongly depend on the energy resolution.

The similar to $\mathrm{Sr}_{2} \mathrm{CuO}_{2} \mathrm{Cl}_{2}$ experimental results were obtained by F. Ronning et al. [20] for $\mathrm{Ca}_{2} \mathrm{CuO}_{2} \mathrm{Cl}_{2}$. The improved spectral quality allows authors to reveal a steep drop in spectral intensity across a contour that is close to the Fermi surface predicted by the band calculation. They concluded that the Fermi surface, which is destroyed by the strong Coulomb interactions, left a remnant in this insulator with a volume and shape similar to what one expects if the strong electron correlation in this system is turned off. The lowest energy peak exhibits a dispersion with approximately the $\left|\cos _{x} a-\cos _{y} a\right|$ form along this remnant Fermi surface, in other words the strong correlation effect deforms this otherwise iso-energetic contour (the non-interacting Fermi surface) into the form that resembles the d-wave like pseudogap dispersion with a very high energy scale of $320 \mathrm{meV}$ [20]. The authors 20] consider the d-wave like dispersion of the parent insulator to be the underlying reason for the pseudogap in the underdoped cuprates. They follow straightforwardly the simplest model approach to ARPES and electronic structure of the photo-hole, and do not consider either alternative approaches, albeit they mention some "parasitic" effects of matrix elements and photon polarization, especially, if these effects hardly keep within their generic model. The approach [20] is demonstrative for many papers on ARPES, in particular, addressing the Fermi surface problem.

At present there are several papers with experimental ARPES data on $\mathrm{Sr}_{2} \mathrm{CuO}_{2} \mathrm{Cl}_{2}$ and $\mathrm{Ca}_{2} \mathrm{CuO}_{2} \mathrm{Cl}_{2}$ systems [5, 6, 9, 21, 22, 20] distinguished by different energy resolution from $25 \mathrm{meV}$ [21] to 105-115 meV [5,6], different photon energy from $10 \mathrm{eV}$ to $80 \mathrm{eV}$ [5,6,23], light polarization [21,23], and the measurement temperature. Despite the many common features there are some important departures. So, contrary to findings 9, the strong "quasiparticle" dispersion was observed in $\mathrm{Sr}_{2} \mathrm{CuO}_{2} \mathrm{Cl}_{2}$ for the $(\pi, 0)$ direction with strong and unusual dependence of the peak amplitude on the light polarization [21]. Kim et al. [22] performed the ARPES measurements in Sr-oxychloride at $150 \mathrm{~K}$, well below the Néel temperature, and have confirmed that the spectrum in the $(\pi, \pi)$ direction consists of a single relatively sharp peak near $(\pi / 2, \pi / 2)$. However, the spectra along $(\pi, 0)$ direction are very broad and consist of at least two peaks separated by about $0.4 \mathrm{eV}$. Just recently, using the ARPES spectra of $\mathrm{Sr}_{2} \mathrm{CuO}_{2} \mathrm{Cl}_{2}$ as an example, the authors [5,6] have experimentally demonstrated a significant impact of electron-photon matrix elements on both the relative spectral intensity and the shape of a low-energy feature in ARPES spectrum.

Many experimental ARPES data and conclusions on $\mathrm{Sr}_{2} \mathrm{CuO}_{2} \mathrm{Cl}_{2}$ known up to now were revised very recently 
in a detailed investigation by C. Dürr et al. [23]. The authors observed the marked oscillating photon-energy dependence of the photoemission signal of the first electron-removal state, which was attributed to the diffraction of the photoelectron wave on the $c$-axis periodically arrayed $\mathrm{CuO}_{2}$ planes. They found a strong polarization dependence in ARPES spectra along the high-symmetry directions, compatible with that expected for a ZhangRice singlet. The ratio between the coherent and incoherent spectral weight in ARPES spectra near the first electron-removal state appears to be photon-energy dependent. Among several mechanisms of this puzzling effect the authors [23] mention the possible contribution of the low-lying electronic states other than the Zhang-Rice singlet.

One of the remarkable spectral features clearly revealed in the ARPES measurements for both insulating cuprates [9.20] is a significant low-energy spectral weight in the $\Gamma(0,0)$ point that accordingly to symmetry of the electricdipole matrix elements implies a significant weight of the purely oxygen odd $e_{u}$-symmetry photo-hole state. However, in the framework of traditional approach one prefers to take no notice of this feature.

Our paper has not for an object the elaboration of the general theory of ARPES in the strongly correlated oxides. The authors would like to emphasize on the possibly simple but real examples an importance of some factors such as matrix element effects, formation of the hole non-adiabatic polaron, often to be disregarded in the framework of conventional approaches to interpretation of ARPES data in copper oxides. At the same time the paper could be considered as a first step in elaboration of an original theory of ARPES in the strongly correlated oxides.

The paper is organised as follows. In Section I we present a brief summary of results of quantum chemical modelling the electronic structure and energy spectrum for the $\mathrm{CuO}_{4}^{6-}$ and $\mathrm{CuO} \mathrm{O}_{4}^{5-}$ centers with one and two holes, respectively. One considers the formation of small and large non-adiabatic polarons. It should be mentioned here that the concept of polarons led to the discovery of the copper oxide superconductors. Section II incorporates an analysis of the generic expression for the photoemission current intensity in the framework of the PJT polaronic approach. In Section III we present a model calculation of dipole matrix elements which describe a transition of a bound $\gamma \mu$ electron to unbound state. An illustrative modelling of the $k$-, and polarization dependence for the matrix element part of the ARPES intensity is given in Section IV.

\section{Electronic structure of the hole state in cuprates. From small to large PJT polaron}

One of the peculiar properties of the doped cuprates is a cross-over from localized to itinerant electronic behaviour with an inhomogeneous distribution of electronic states which description in a wide range of compositions from a single-valent antiferromagnetic insulator to a mixed valent bad metal is a fundamental problem of the solid state physics.

Unfortunately, at present there is no a general consensus concerning the nature of valent electron and hole states which form optical and electron (PES, XPS, EELS) spectra even in insulating cuprates like $\mathrm{La}_{2} \mathrm{CuO}_{4}, \mathrm{YBa}_{2} \mathrm{Cu}_{3} \mathrm{O}_{6}$, $\mathrm{Sr}_{2} \mathrm{CuO}_{2} \mathrm{Cl}_{2}$. It is becoming increasingly difficult to reconcile experimental results with the expectations of the simple band models and Fermi liquid theory.

One of the firmly established facts relates the $\mathrm{CuO}$ plane character of the valent states with the $b_{1 g}\left(d_{x 2-y_{2}}\right)$ hole ground state for the $\mathrm{CuO}_{4}^{6-}$ center. Various spectroscopic methods link the low-energy excitations in the range $E \geq 2 \mathrm{eV}$ to the charge transfer $\mathrm{O} 2 p \rightarrow C u 3 d$ within the $\mathrm{CuO}$ plane. At a first glance, optical and electron spectra manifest both localised and delocalised, or band character of electronic states. Lack of reliable interpretation of optical and electron spectra in strongly correlated oxides appears to be a result of difficulties in theoretical description of strong covalency and strong intracenter and intercenter correlations.

The parent cuprates such as $\mathrm{CuO}, \mathrm{Sr}_{2} \mathrm{CuO}_{2} \mathrm{Cl}_{2}, \mathrm{La}_{2} \mathrm{CuO}_{4}, \mathrm{YBa}_{2} \mathrm{Cu}_{3} \mathrm{O}_{6}$ provide typical examples of systems with strong local correlations when dielectric antiferromagnetic ground state is mainly specified by potential energy of electron-electron coupling. Kinetic energy would prefer formation of half-filled band with typically metallic behavior. Occurrence of the dielectric antiferromagnetic phase of the parent cuprates itself and wide opportunities of the interacting $\mathrm{CuO}_{4}$-centers model in explanation of many optical and electron spectra for various copper oxides could be considered to be a convincing evidence in favor of quasiparticle states localised predominantly on the $\mathrm{CuO}_{4}$-clusters which do not obey conventional band model description. This is a common place as for $\mathrm{Cu} 3 d-O 2 p$-hybrid states, but purely oxygen non-bonding states are currently described as the band ones. Such a discrimination is partially based on a rather simplified relation between correlation and Slater electrostatic integrals like $F_{0}(3 d 3 d)>F_{0}(2 p 2 p)$, and typical, for instance, for the Anderson impurity model.

However, correlation effects appear to be of particular importance for oxygen hole states which was pointed out by Hirsch et al. 24] in their theory of "anionic metal". A simple model for localisation of the oxygen holes and some other puzzling consequences of conjectured [24] multiplet structure for anionic background were considered recently [28]. A strongly correlated behaviour of the $O 2 p$-holes to some extent gives them equal rights with $3 d$-holes and leads to reconsideration of many conventional approaches and models which ignore strong $O 2 p$-correlations, for instance, the Anderson impurity model which considers purely oxygen states to be band-like.

So, a quantum-chemical cluster approach appears to be more relevant for description of strongly correlated oxides as it allows to account for correlation effects both for cation and anion states by the most optimal manner. It seems, the parent copper oxides can be treated by a conventional quantum-chemical ligand-field theory, so a 
localized description can be considered to be a relevant starting point for modelling the electronic structure both in ground and excited states.

First of all, the modelling implies a choice of the effective basis set of atomic orbitals, such as $\mathrm{Cu} 3 \mathrm{~d}$ - and $\mathrm{O} 2 \mathrm{p}$ orbitals in the cuprates. Then we introduce the $\mathrm{CuO}_{4}$ center being the minimal atomic cluster that posesses the relevant point symmetry $D_{4 h}$ and incorporates the main $C u 3 d-O 2 p$ covalent bond. This center could be considered as an elementary building block to effectively model the electronic structure both in ground and excited states. Along with the symmetry and covalency the $\mathrm{CuO}_{4}$ center allows to account for the electronic and vibronic correlations.

\subsection{Electronic structure and energy spectrum for the $\mathrm{CuO}_{4}^{6-}$ and $\mathrm{CuO}_{4}^{5-}$ centers in cuprates}

Slightly distorted tetragonal $\mathrm{CuO}_{4}$ center is the only common cell of crystalline and electronic structure in a wide variety of high- $T_{c}$ copper oxides and related parent systems. Restricted atomic basis of five $C u 3 d$ and twelve $\mathrm{O} 2 \mathrm{p}$ atomic orbitals for the $\mathrm{CuO}_{4}$ cluster with $D_{4 h}$ symmetry yields seventeen symmetrised orbitals with $a_{1 g}, a_{2 g}$, $b_{1 g}, b_{2 g}, e_{g}$ (even - gerade) and $a_{2 u}, b_{2 u}, e_{u}$ (odd - ungerade) symmetry. The even $C u 3 d$ orbitals with $a_{1 g}\left(3 d_{z^{2}}\right)$, $b_{1 g}\left(3 d_{x^{2}-y^{2}}\right), b_{2 g}\left(3 d_{x y}\right), e_{g}\left(3 d_{x z}, 3 d_{y z}\right)$ symmetry hybridize with even $O 2 p$ orbitals with the same symmetry thus forming the appropriate bonding $\gamma^{b}$ and antibonding $\gamma^{a}$ molecular orbitals. Among the odd orbitals only $e_{u}(\sigma)$ and $e_{u}(\pi)$ ones hybridize each other due to a strong $O 2 p-O 2 p$ coupling to form bonding and antibonding purely oxygen molecular orbitals $e_{u}^{b}$ and $e_{u}^{a}$, respectively. Purely oxygen $a_{2 g}, a_{2 u}, b_{2 u}$ orbitals are nonbonding. All the plane molecular orbitals could be subdivided to $\sigma\left(a_{1 g}, b_{1 g}, e_{u}(\sigma)\right)$ and $\pi\left(a_{2 g}, b_{2 g}, e_{u}(\pi)\right)$ ones, depending on the orientation of $O 2 p$ orbitals.

Quantum-chemical cluster approach allows the optimal account for electrostatic correlations and description of a rather complex electronic structure of the two-hole $\mathrm{CuO}_{4}^{5-}$ center. Firstly, one should note an importance of configurational interaction, which could be rather simply illustrated by the model cluster calculation [29]. Indeed, the wave function and energy of the ground state term ${ }^{1} A_{1 g}$ (Zhang-Rice singlet) exhibits an essential coupling for three configurations like $b_{1 g} b_{1 g}$ :

$$
\left|{ }^{1} A_{1 g}>=0.82\right|\left(b_{1 g}^{b}\right)^{2}>+0.55\left|b_{1 g}^{a} b_{1 g}^{b}>-0.16\right|\left(b_{1 g}^{a}\right)^{2}>.
$$

If to consider the ${ }^{1} A_{1 g}$ singlet originating from the only $\left(b_{1 g}^{b}\right)^{2}$ configuration and to represent its energy as follows

$$
E\left({ }^{1} A_{1 g}\right)=2 \varepsilon\left(b_{1 g}^{a}\right)+U+\Delta U
$$

with $U$ being the contribution of the hole-hole $b_{1 g}^{b}-b_{1 g}^{b}$ interaction, and $\Delta U$ that of configurational interaction, then

$$
U+\Delta U=4.7-3.5=1.2(\mathrm{eV})
$$

which value is puzzlingly small as compared with the bare value $U_{d}=A+4 B+3 C \simeq 10 \mathrm{eV}$ for purely atomic $d_{x^{2}-y^{2}}^{2}$ configuration.

A concept of the well isolated Zhang-Rice singlet to be a ground state of the two-hole $\mathrm{CuO}_{4}^{5-}$ center, is a guideline of many popular model approaches [33]. Namely with ZR singlet one associates the first electron-removal state and the lowest-lying features in ARPES spectra. At the same time, a number of experimental data and theoretical models evidence a more complicated structure of the valent multiplet for the two-hole $\mathrm{CuO} \mathrm{O}_{4}^{5-}$ center.

A model of the valent ${ }^{1} A_{1 g}-{ }^{1} E_{u}$ multiplet, developed in Refs. [29, 34, 35], implies a quasi-degeneracy in the ground state of the two-hole $C u O_{4}^{5-}$ center with two close in energy ${ }^{1} A_{1 g}$ and ${ }^{1} E_{u}$ terms of $b_{1 g}^{2}$ and $b_{1 g} e_{u}$ configurations, respectively. In other words, one implies two near equivalent allocations for the additional hole, either to the $C u 3 d O 2 p$ hybrid $b_{1 g}$ state, or to purely oxygen $e_{u}$ state with peculiar $C u^{2+}-C u^{3+}$ valence resonance (see Fig.1). Occurrence of the localized purely oxygen $e_{u}$ like states is provided, in particular, by the specific properties of the "non-rigid" anionic $O 2 p^{6}$ background [24,28].

The model approach under consideration is based both on microscopic quantum chemical study of the model copper-oxygen clusters 29 31] and a large variety of experimental data. To the best of our knowledge one of the first quantitative conclusions on the competitive role of the hybrid copper-oxygen $b_{1 g}\left(d_{x^{2}-y^{2}}\right)$ orbital and purely oxygen $\mathrm{O} 2 p_{\pi}$ orbitals in formation of valent states near the Fermi level in the $\mathrm{CuO}$ planes has been made by Jiro Tanaka et al. [30] (see also more later publication [31]).

In a sense, the valent $\left(b_{1 g}^{2}\right)^{1} A_{1 g}-\left(b_{1 g} e_{u}\right)^{1} E_{u}$ manifold for the hole $C u O_{4}^{5-}$ center implies an unconventional state with $\mathrm{Cu}$ valence resonating between $\mathrm{Cu}^{3+}$ and $\mathrm{Cu}^{2+}$, or "ionic-covalent" bonding [32]. In fact, the $\mathrm{CuO}$ center with the valent $\left(b_{1 g}^{2}\right)^{1} A_{1 g}-\left(b_{1 g} e_{u}\right)^{1} E_{u}$ manifold represents a specific version of the "correlation" polaron, introduced by Goodenough and Zhou [32].

The model allows to consistently explain many puzzling properties both of insulating and superconducting cuprates: the mid-infrared (MIR) region absorption bands [29], the (pseudo)Jahn-Teller effect and related phenomena [34], the spin properties [35].

The presence of small polarons in semiconducting copper oxides has been detected with photoinduced infrared absorption measurements, infrared spectroscopy, and X-ray absorption fine structure techniques. 
One of the most exciting experimental evidences in favour of the model with the valent ${ }^{1} A_{1 g}-{ }^{1} E_{u}$ multiplet is associated with observation in the doped cuprates of the MIR bands which polarisation features are compatible with those for ${ }^{1} A_{1 g}-{ }^{1} E_{u}$ intra-multiplet dipole transitions [29]. The corresponding transition energies observed for various cuprates are of the order of a few tenths of $\mathrm{eV}$, that exhibits a typical energy scale for the valent multiplet.

The $e_{u}$ hole can be coupled with the $b_{1 g}$ hole both antiferro- and ferromagnetically. This a rather simple consideration indicates clearly a neccessity to incorporate in valent multiplet both spin-singlet $\left(b_{1 g} e_{u}\right)^{1} E_{u}$ and spintriplet $\left(b_{1 g} e_{u}\right)^{3} E_{u}$, which energy could be even lower due to ferromagnetic $b_{1 g}-e_{u}$ exchange. Indeed, the low-lying spin-triplet state for the two-hole $\mathrm{CuO}_{4}^{5-}$ center was revealed with the help of ${ }^{63,65} \mathrm{Cu} \mathrm{NQR}^{-}$in $\mathrm{La}_{2} \mathrm{Cu}_{0.5} \mathrm{Li}_{0.5} \mathrm{O}_{4}$ with singlet-triplet separation $\Delta_{S T}=0.13 \mathrm{eV}[36]$. Indirect manifestation of $O 2 p \pi$, or $e_{u}$ valent states were detected in Knight shift measurements by NMR for $123-\mathrm{YBaCuO}$ system [37]. In connection with the valent ${ }^{1} A_{1 g}-{ }^{1} E_{u}$ multiplet model for copper oxides one should note and comment the results of paper by Tjeng et al. 38., where the authors state that they "are able to unravel the different spin states in the single-particle excitation spectrum of antiferromagnetic $\mathrm{CuO}$ and show that the top of the valence band is of pure singlet character, which provides strong support for the existence and stability of Zhang-Rice singlets in high- $T_{c}$ cuprates". However, in their photoemission work they made use of the $C u 2 p_{3 / 2}\left(L_{3}\right)$ resonance condition that allows to detect unambiguously only copper photohole states, hence they cannot see the purely oxygen photohole $e_{u}$ states.

It should be noted that the complicated ${ }^{1} A_{1 g}-{ }^{1,3} E_{u}$ structure of the valent multiplet for the two-hole $C u O_{4}^{5-}$ center has to be revealed in the photoemission spectra, all the more that the odd ${ }^{1,3} E_{u}$ terms play a principal role: namely these yield a nonzero contribution to ARPES for $\mathbf{k}=0$, or, in other words, at $\Gamma$ point. In this connection one should note experimental measurements of the photoemission spectra in $\mathrm{Sr}_{2} \mathrm{CuO}_{2} \mathrm{Cl}_{2}$ [9] and $\mathrm{Ca}_{2} \mathrm{CuO}_{2} \mathrm{Cl}_{2}$ [20]. All these clearly detect a nonzero photocurrent intensity in the BZ center, thus supporting the ${ }^{1} A_{1 g}-{ }^{1,3} E_{u}$ structure of the ground state valent multiplet.

\subsection{Effective vibronic Hamiltonian and small (pseudo)Jahn-Teller polaron}

The orbital near-degeneracy leads to strong electron-lattice (vibronic) effects. For $\left({ }^{1} A_{1 g},{ }^{1} E_{u}\right)$ valent multiplet in the $\mathrm{CuO}_{4}$-center one has to consider the active $Q_{\gamma}$ nuclear displacement modes with $\gamma=a_{1 g}, b_{1 g}, b_{2 g}, e_{u}$. Effective vibronic Hamiltonian $\hat{H}_{v i b r}$ is a sum of two contributions

$$
\hat{H}_{v i b r}=\hat{H}_{E E}+\hat{H}_{A E}
$$

that of vibronic coupling within the ${ }^{1} E_{u}$ term, and as well the vibronic coupling of the ${ }^{1} E_{u}$ and ${ }^{1} A_{1 g}$ terms, respectively. Vibronic Hamiltonian for isolated ${ }^{1} E_{u}$ term is familiar for a so called $E-b_{1}-b_{2}$ problem [26], and in terms of orbital operators $\hat{V}_{\gamma}\left(\gamma=a_{1 g}, b_{1 g}, b_{2 g}, e_{u}\right)$ has a following form:

$$
\hat{H}_{E E}=v_{b_{1 g}} \hat{V}_{b_{1 g}} Q_{b_{1 g}}+v_{b_{2 g}} \hat{V}_{b_{2 g}} Q_{b_{2 g}}
$$

with $v_{b_{1 g}, b_{2 g}}$ being vibronic coupling parameters. Vibronic Hamiltonian $\hat{H}_{A E}$ contains two terms

$$
\hat{H}_{A E}=v_{a_{1 g}} \hat{V}_{a_{1 g}} Q_{a_{1 g}}+\sum_{e_{u}} v_{e}\left(\vec{Q}_{e_{u}}^{i} \hat{\vec{V}}_{e_{u}}\right) .
$$

The first provides the connection between the $A-E$ separation and symmetrical $Q_{a_{1 g}}$ mode, while the second describes a linear vibronic coupling of the ${ }^{1} E_{u}$ and ${ }^{1} A_{1 g}$ terms due to the active odd $Q_{e_{u}^{x}}, Q_{e_{u}^{y}}$ modes. One should note that for the $\mathrm{CuO}_{4}$ center one exists three types of the $e_{u}$ modes. Vibronic Hamiltonian $\hat{H}_{v i b}$ has to be added by elastic energy $\hat{U}_{Q}$ for the $\mathrm{CuO}_{4}$ center

$$
\hat{U}(Q)=\sum_{i} \frac{\omega_{i}^{2} Q_{i}^{2}}{2}
$$

where summing runs over all normal displacements modes. A rather complete examination of the (pseudo)JahnTeller $\left({ }^{1} A_{1 g},{ }^{1} E_{u}\right)-a_{1 g}-b_{1 g}-b_{2 g}-e_{u}$ problem was carried out in Ref. 34. Depending on the relation between vibronic and elastic parameters one finds to be three situations:

1. Weak vibronic coupling when $\hat{H}_{v i b}$ results only in a rather small renormalization of bare elastic constants.

2. The Jahn-Teller effect for the $E_{u}$ term $\left(E-b_{1}-b_{2}\right.$ problem) with emergence of two-well adiabatic potential for the rhombic modes with either $b_{1 g}$, or $b_{2 g}$ symmetry.

3. Strong pseudo-Jahn-Teller effect with vibronic mixing of $\left({ }^{1} A_{1 g}\right.$ and $\left.{ }^{1} E_{u}\right)$ terms and emergence of four-well adiabatic potential.

In practice one might observe various manifestations of the (pseudo)Jahn-Teller effect including the conventional static or dynamic effect, local structural instability, including the dipole one, spontaneous and induced local structural phase transitions with reconstruction of the multi-well adiabatic potential.

The presence of the PJT centers, or small PJT polarons in semiconducting copper oxides has been detected, in particular, with photoinduced infrared absorption measurements, infrared spectroscopy, and X-ray absorption fine 
structure techniques. The photoinduced infrared absorption measurements on a number of insulating copper oxides have unambiguously revealed the formation of a localized electronic state accompanied by a localized structural distortion. These two aspects of the data have demonstrated the self-localized polaronic nature of the photo-injected carriers with a complex optical response spreading on a rather wide spectral range $0.1 \div 1.0 \mathrm{eV}$.

\subsection{Large non-adiabatic hole polarons in the $\mathrm{CuO}_{2}$ layers of cuprates}

Non-zero hole transfer together with strong intercenter coupling due to the common oxygen favours the transformation of the small PJT polaron into a large non-adiabatic PJT polaron. Stabilization of a large polaron causes the hole to be shared over several $\mathrm{CuO}_{4}$ centers that could result in an optimal relaxation of the elastic energy and vibronic coupling all over the polaron volume.

A model of large non-adiabatic hole polarons for the doped copper oxides was proposed by Bersuker and Goodenough [25]. The large polaron could contain a hole cloud distributed over a significant number of the $\mathrm{CuO}_{4}$ centers. So, the authors 25 considered polarons containing 5 to $6 \mathrm{CuO}_{4}$ centers.

One of the model approaches capable to effectively describe such a polaron implies an extention of the conventional PJT problem with choice of a set of the symmetrized quasimolecular orbitals constructed from the valent hole states localized on the $\mathrm{CuO}_{4}$ clusters involved in the PJT polaron (similar to the known LCAO-method), and a set of symmetrized displacements for the corresponding copper and oxygen atoms.

The symmetrized quasimolecular orbitals can be build as linear combinations of the valent hole molecular orbitals $\psi_{\gamma \mu}(\mathbf{r})$ for the $\mathrm{CuO}_{4}$ cluster

$$
\Psi_{\Gamma M}^{(\gamma \mu)}(\mathbf{R})=\sum_{\mathbf{r}} C_{\gamma \mu}^{\Gamma M}(\mathbf{R}+\mathbf{r}) \psi_{\gamma \mu}(\mathbf{R}+\mathbf{r}) \psi_{0}(\mathbf{R}+\mathbf{r})
$$

where $\psi_{0}(\mathbf{r})$ is ground state wave function of the $C u O_{4}^{6-}$ cluster, $\Gamma M, \gamma \mu$ are irreducible representations of the point symmetry group, $C_{\gamma \mu}^{\Gamma M}$ are the symmetry coefficients, $\mathbf{R}$ is a radius-vector of the polaronic center of symmetry, and the sum runs over all the $\mathrm{CuO}_{4}$ centers inside large polaron. For illustration we present the symmetrized quasimolecular orbitals for the large polaron containing $5 \mathrm{CuO}$ centers (see Fig.2) with valent $b_{1 g}$ orbital:

$$
\begin{gathered}
\Psi_{A_{1 g}}^{\left(b_{1 g}\right)}=\chi(\mathbf{0}), \Psi_{A_{1 g}^{\prime}}^{\left(b_{1 g}\right)}=\frac{1}{2}\{\chi(\mathbf{x})+\chi(-\mathbf{x})+\chi(\mathbf{y})+\chi(-\mathbf{y})\} \\
\Psi_{B_{1 g}}^{\left(b_{1 g}\right)}=\frac{1}{2}\{\chi(\mathbf{x})+\chi(-\mathbf{x})-\chi(\mathbf{y})-\chi(-\mathbf{y})\} \\
\Psi_{E_{u 1}}^{\left(b_{1 g}\right)}=\frac{1}{2}\{\chi(\mathbf{x})-\chi(-\mathbf{x})+\chi(\mathbf{y})-\chi(-\mathbf{y})\} \\
\Psi_{E_{u 2}}^{\left(b_{1 g}\right)}=\frac{1}{2}\{-\chi(\mathbf{x})+\chi(-\mathbf{x})+\chi(\mathbf{y})-\chi(-\mathbf{y})\}
\end{gathered}
$$

where $|\chi\rangle=\left|\psi_{b_{1 g}} \psi_{b_{1 g}}\right\rangle \equiv\left|\psi_{0} \psi_{0}\right\rangle$ is the Zhang-Rice singlet wave function.

Thus, we obtain a polaronic $A_{1}^{1}-A_{1}^{2}-B_{1}-E$ manifold generated by the $b_{1 g}$-state of the $C u O_{4}$ centers. This manifold incorporates two quasimolecular orbitals with $s$-symmetry, one with $d_{x 2-y 2}$-symmetry, and doublet with $p_{x, y}$-symmetry. The account for the hole transfer results in a bare splitting within the manifold. The orbital states with the same symmetry will mix forming the superpositions

$$
\Phi_{\Gamma M}(\mathbf{R})=\sum_{\gamma \mu} \sum_{\mathbf{r}} A_{\Gamma M}^{(\gamma \mu)} C_{\gamma \mu}^{\Gamma M}(\mathbf{R}+\mathbf{r}) \psi_{\gamma \mu}(\mathbf{R}+\mathbf{r}) \psi_{0}(\mathbf{R}+\mathbf{r}) .
$$

One should note a specific bare quasimolecular orbitals for the $N$-center "molecule" generated by local current states like $e_{u \pm} \propto\left(e_{u x} \pm i e_{u y}\right)$. These manifest the $N$-fold pattern of microscopic circulating currents resulting both in a state with nonzero magnetic moment and in a state with zero magnetic moment. In the latter case the time-reversal symmetry as well as rotational symmetry is broken but the product of the two is conserved.

For many applications in scattering problems it is useful to introduce the form-factors

$$
f_{\Gamma M}^{(\gamma \mu)}(\mathbf{k})=\sum_{\mathbf{r}} A_{\Gamma M}^{(\gamma \mu)} C_{\gamma \mu}^{\Gamma M}(\mathbf{r}) e^{i \mathbf{k r}}
$$

to describe the spatial distribution of the hole density inside a large polaron.

Some form-factors for $N=4$ large polaron (see Fig.2) are

$$
f_{B_{1 g}}^{\left(b_{1 g}\right)}(\mathbf{k})=2 \cos \left(\frac{a k_{x}}{2}\right) \cos \left(\frac{a k_{y}}{2}\right),
$$




$$
\begin{gathered}
f_{B_{1 g}}^{\left(e_{u} x\right)}(\mathbf{k})=-i \sqrt{2} \sin \left(\frac{a k_{x}}{2}\right) \cos \left(\frac{a k_{y}}{2}\right), \quad f_{B_{1 g}}^{\left(e_{u} y\right)}(\mathbf{k})=i \sqrt{2} \cos \left(\frac{a k_{x}}{2}\right) \sin \left(\frac{a k_{y}}{2}\right), \\
f_{E_{u} x}^{\left(b_{1 g}\right)}(\mathbf{k})=2 i \sin \left(\frac{a k_{x}}{2}\right) \cos \left(\frac{a k_{y}}{2}\right), \quad f_{E_{u} y}^{\left(b_{1 g}\right)}(\mathbf{k})=2 i \sqrt{2} \cos \left(\frac{a k_{x}}{2}\right) \sin \left(\frac{a k_{y}}{2}\right), \\
f_{E_{u} x}^{e_{u} x}(\mathbf{k})=f_{E_{u} y}^{e_{u} y}(\mathbf{k})=2 \cos \left(\frac{a k_{x}}{2}\right) \cos \left(\frac{a k_{y}}{2}\right), \quad f_{E_{u} x}^{e_{u} y}(\mathbf{k})=f_{E_{u} x}^{e_{u} y}(\mathbf{k})=0 .
\end{gathered}
$$

A set of the symmetrized quasimolecular orbitals $\Phi_{\Gamma M}$ and symmetrized displacements $Q_{\Gamma M}$ for the copper and oxygen atoms inside large polaron form a basis for a rather standard, albeit complicated, vibronic PJT problem which solution gives an energy spectrum and appropriate wave functions $\Psi_{\alpha \Gamma n}$ describing strongly correlated vibronic nature of the polaronic states. Here, $\alpha \Gamma n$ represent a set of quantum numbers which define a vibronic state. It should be noted, that only given the extremely simplifying assumptions this function could be written in a familiar Born-Oppenheimer form like $\Psi_{\alpha \Gamma n}=\Phi_{\Gamma M} \chi_{\alpha \Gamma n}(Q)$, where $\chi_{\alpha \Gamma n}(Q)$ is a vibrational function. In a wide sense, excitation spectrum of the large PJT polaron involves a whole vibronic spectrum originated from quasimolecular $(\gamma) \Gamma$ multiplet. It should be noted that the symmetry classification of polaronic hole states allows to elucidate some similarities with atomic system and partial waves; one might say about $s-, p-, d-, \ldots$ like hole states. One should note an occurrence of the time-reversal symmetry breaking current states for the large PJT polaron. In addition, we must emphasize one more the specific role of the near-degeneracy for the valent manifold, and probable PJT effect, for the individual $\mathrm{CuO}_{4}$ center in formation of a large non-adiabatic polaron.

Finally, large PJT-polaron in the lattice could be represented as a system of the $\mathrm{CuO}_{4}$ centers with a set of metastable states $\Psi_{\alpha \Gamma n}$ specified by a binding energy and a life-time. Its nature implies strong charge fluctuations, so, it seems rather difficult to confine such a polaron within a single $\mathrm{CuO}_{2}$ layer. In other words, the large PJT polaronic nature of photo-hole implies its $3 D$ structure with finite dimension, or correlation length, in the $c$-direction. Three-dimensional structure of the large PJT polaron could result in a rather strong $k_{\perp}$, and consequently, photon-energy dependence of the ARPES intensity. Nevertheless, below we restrict ourselves, for simplicity, with the planar PJT polarons.

In practice, for real systems the polaron will effectively couple with all the phonon modes which are active in the PJT effect [26]. In other words, a large non-adiabatic PJT polaron may be considered as a bounded state of the large PJT-center and phonons. Coupling with phonons and spin system will result in an effective enlargement of polaron. The simplest way to account for this effect implies the introducing of a momentum-dependent cut-off factor to a polaronic form-factor. In addition, the phonon system will determine the relaxation dynamics of polaron states, and gives rise an effective dispersion to the hole spectral function.

It should be noted that the quasiparticle behavior of polaron implies, as a rule, a single rather strongly bounded long-lived term. For this one can introduce an effective Hamiltonian which should include polaronic transport and coupling to phonon and spin lattice modes thus providing the coherent and incoherent part of the quasiparticle spectral function. It should be noted that the effective quasiparticle Hamiltonian could be look like as familiar Hubbard, or $t-J$ Hamiltonian. The remaining short-lived polaronic states will give rise to a rather wide structureless and dispersionless background in spectral region of valent manifold.

Above we have considered the large lattice polaron and ignored the role of the antiferromagnetic background and appropriate spin fluctuations. Many authors have considered the formation of magnetic (spin) polarons [27, moreover the assumption of spin-polaronic nature of the photo-hole, described in the framework of the extended $t-J$ model is one of the most popular approaches to the interpretation of ARPES data in cuprates. So-called electron-hole asymmetric small polarons were introduced by J.E. Hirsch [24]. In general, the polarons must be of complex spin-lattice hybrid type with a complicated spatial distribution of the electron and spin densities, and local structure distortions. 


\section{Expression for photointensity}

Below we consider an expression for intensity of the photoemission with creation of immobile hole PJT polaron. An effective Hamiltonian for interaction with the electromagnetic field of frequency $\omega$ and polarisation e could be written within a polaronic manifold as follows

$$
\hat{H}_{i n t}=\sum_{\Gamma M} \sum_{\mathbf{k}} \mathcal{M}_{\Gamma M}(\mathbf{k}, \mathbf{e}) \hat{c}_{\mathbf{k} \sigma}^{\dagger} \hat{h}_{\Gamma M \sigma}^{\dagger}+H . c .,
$$

where $\mathbf{k}$ is a momentum of the final state of the photoelectron registered by the detector, $\hat{c}_{\mathbf{k} \sigma}^{\dagger}$ and $\hat{h}_{\Gamma M \sigma}^{\dagger}$ are creation operators for photoelectron and photohole, respectively. The matrix element is given by

$$
\mathcal{M}_{\Gamma M}(\mathbf{k}, \mathbf{e})=\left\langle\psi_{\mathbf{k}}(\mathbf{r}) \Psi_{\Gamma M}^{(N-1)}\left|\hat{H}_{e R}\right| \Psi_{g}^{(N)}\right\rangle,
$$

where

$$
\hat{H}_{e R}=\frac{e \hbar}{2 m c}(\mathbf{p} \cdot \mathbf{A}+\mathbf{A} \cdot \mathbf{p})
$$

is the interaction Hamiltonian with the electron momentum operator $\mathbf{p}$ and the vector potential $\mathbf{A}$ of the photon field; $\Psi_{g}^{(N)}$ is the wave function for the ground state; $\Psi_{\Gamma M}^{(N-1)}$ is the wave function for a $\Gamma M$ state with one removed electron (one additional hole); $\psi_{\mathbf{k}}(\mathbf{r})$ is the photoelectron wave function. It should be noted that expression (9) already implies a number of noticeable simplifications.

Modelling the photoelectron wave function by a plane wave, we rewrite the expression for the matrix element (9) as follows

$$
\mathcal{M}_{\Gamma M}(\mathbf{k}, \mathbf{e})=\sum_{\gamma \mu} f_{\Gamma M}^{(\gamma \mu)}(\mathbf{k}) M_{\gamma \mu}(\mathbf{k}, \mathbf{e})
$$

where in the dipole approximation

$$
M_{\gamma \mu}(\mathbf{k}, \mathbf{e})=\left\langle\psi_{\gamma \mu}(\mathbf{r})|(\mathbf{e} \cdot \mathbf{r})| e^{i \mathbf{k r}}\right\rangle .
$$

Finally, the expression for the photoemission intensity may be transformed into

$$
I(\mathbf{k}, \omega, \mathbf{e}) \propto \sum_{\Gamma_{1} M_{1} ; \Gamma_{2} M_{2}} \mathcal{M}_{\Gamma_{1} M_{1}}^{*}(\mathbf{k}, \mathbf{e}) \mathcal{M}_{\Gamma_{2} M_{2}}(\mathbf{k}, \mathbf{e}) A_{\Gamma_{1} M_{1} ; \Gamma_{2} M_{2}}(\omega),
$$

where the ground $|g\rangle$ and excited $|e\rangle$ states are the nonperturbed electron-vibrational states for the N-center cluster and vibronic ones for the hole PJT polaron, respectively. Emission spectral functions have a quite standard form

$$
\begin{aligned}
A_{\Gamma_{1} M_{1} ; \Gamma_{2} M_{2}}(\omega)= & \frac{1}{2} \sum_{\sigma, e, g} e^{-\beta E_{g}}\left\langle e\left|\hat{h}_{\Gamma_{1} M_{1} \sigma}^{\dagger}\right| g\right\rangle\left\langle g\left|\hat{h}_{\Gamma_{2} M_{2} \sigma}\right| e\right\rangle \delta\left(\omega+E_{e}-E_{g}\right)= \\
& \frac{1}{2} \sum_{\sigma} \int d t e^{i \omega t}\left\langle\hat{h}_{\Gamma_{1} M_{1} \sigma}^{\dagger}(t) \hat{h}_{\Gamma_{2} M_{2} \sigma}(0)\right\rangle .
\end{aligned}
$$

Spectral functions contain a complete information about complex vibronic structure of the PJT polaron, and describe both the partial $\Gamma$-contributions at $\Gamma_{1}=\Gamma_{2}$ and interference effects for different states with the same symmetry. These obey the sum rules

$$
\int \frac{d \omega}{2 \pi} A_{\Gamma_{1} M_{1} ; \Gamma_{2} M_{2}}(\omega)=n_{\Gamma_{1} M_{1}} \delta_{\Gamma_{1} M_{1} ; \Gamma_{2} M_{2}} .
$$

Despite the extremely rough simplifying approximations Exp.(12) displays very complex multi-level structure for photo-intensity with nontrivial polarization and $\mathbf{k}, \omega$ dependence.

Calculation of spectral functions $A_{\Gamma_{1} M_{1} ; \Gamma_{2} M_{2}}(\omega)$ for the large PJT polaron represents an extremely complex problem even at very strong simplifications [26]. For illustration, one might refer to a similar problem with spectral function which describes the line-shape of the optical $A-E$ transition between the orbital singlet and orbital JT doublet [26].

It should be noted, that, in a sense, the non-diagonal spectral functions $A_{\Gamma_{1} M_{1} ; \Gamma_{2} M_{2}}(\omega)$ describe the spectral weight transfer between $\Gamma_{1}$ and $\Gamma_{2}$ bands. In a whole, the PJT polaronic nature of the photo-hole provides a k-dependent ARPES spectral line-shape, in particular, with dispersive peak position.

\section{One-electron matrix element}




\subsection{Copper contribution}

The copper atomic orbital with symmetry $\gamma \mu$ can be represented in the form

$$
d_{\gamma \mu}(\mathbf{r})=R_{3 d}(r) \sum_{m} \alpha_{2 m}(\gamma \mu) Y_{2 m}(\mathbf{r}),
$$

where $\alpha_{2 m}(\gamma \mu)$ are coefficients specified by the symmetry requirements, $R_{3 d}(r)$ radial wave function, which we assume to be of simple Slater form

$$
R_{3 d}(r)=\frac{2}{81} \sqrt{\frac{2}{15}} \frac{r^{2}}{a_{d}^{3} \sqrt{a_{d}}} \exp \left\{-\frac{r}{3 a_{d}}\right\} .
$$

Inserting these expressions to (11) and making use the familiar expansion for the plane wave 40]

$$
e^{i \mathbf{k r}}=4 \pi \sum_{L=0}^{\infty} \sum_{M=-L}^{L} i^{L} j_{L}(k r) Y_{L M}^{*}(\mathbf{k}) Y_{L M}(\mathbf{r})
$$

we rewrite (11) as follows

$$
\begin{gathered}
M_{\gamma \mu}^{(C u)}(\mathbf{k}, \mathbf{e})=\left\langle d_{\gamma \mu}(\mathbf{r})|(\mathbf{e} \cdot \mathbf{r})| e^{i \mathbf{k r}}\right\rangle= \\
\frac{4 \pi i}{\sqrt{5}}\left\{\sqrt{2} D_{1}(k) K_{1}^{(\gamma \mu)}(\mathbf{e}, \mathbf{k})+\sqrt{3} D_{3}(k) K_{3}^{(\gamma \mu)}(\mathbf{e}, \mathbf{k})\right\}
\end{gathered}
$$

where we denote

$$
\begin{gathered}
D_{1}(k)=\left\langle R_{3 d}(r)|r| j_{1}(k r)\right\rangle=864 \sqrt{\frac{6}{5}} \frac{a_{d}^{3} \sqrt{a_{d}} k\left(5-27 a_{d}^{2} k^{2}\right)}{\left(1+9 a_{d}^{2} k^{2}\right)^{5}}, \\
D_{3}(k)=\left\langle R_{3 d}(r)|r| j_{3}(k r)\right\rangle=62208 \sqrt{\frac{6}{5}} \frac{a_{d}^{5} \sqrt{a_{d}} k^{3}}{\left(1+9 a_{d}^{2} k^{2}\right)^{5}}, \\
K_{L}^{(\gamma \mu)}(\mathbf{e}, \mathbf{k})=\left[Y^{L} \times e^{1}\right]^{2 \gamma \mu *}=\sum_{M, q, m}(-1)^{q} e_{-q} C_{L M 1 q}^{2 m} Y_{L M}^{*}(\mathbf{k}) \alpha_{2 m}^{*}(\gamma \mu) .
\end{gathered}
$$

Here $C_{L M 1-q}^{2 m}$ are the Clebsch-Gordan coefficients. The photoelectron energy dependence of quantities $D_{1}(k)$ and $D_{3}(k)$ given the $C u 3 d$ radial parameter $a_{d}=0.35 \AA$ is shown in Fig.3.

\subsection{Oxygen contribution}

The oxygen molecular orbital can be represented as a linear combination of atomic $O 2 p$ functions centered at appropriate oxygen positions

$$
p_{\gamma \mu}(\mathbf{r})=\sum_{\mathbf{t} m} C_{m}^{\gamma \mu}(\mathbf{t}) R_{2 p}(|\mathbf{r}-\mathbf{t}|) Y_{1 m}(\mathbf{r}-\mathbf{t})
$$

where $C_{m}^{\gamma \mu}(\mathbf{t})$ are coefficients specified by the symmetry requirements, $R_{2 p}(r)$ radial wave function. Inserting (17) to (11) and making substitution $\mathbf{r}^{\prime}=\mathbf{r}-\mathbf{t}$, we reduce (11) to

$$
\begin{aligned}
M(\mathbf{k}, \mathbf{e}) & =\sum_{\mathbf{t} m} C_{m}^{*}(\mathbf{t})(\mathbf{e} \cdot \mathbf{t}) e^{i \mathbf{k t}}\left\langle R_{2 p}\left(\mathbf{r}^{\prime}\right) Y_{1 m}\left(\mathbf{r}^{\prime}\right) \mid e^{i \mathbf{k} \mathbf{r}^{\prime}}\right\rangle \\
& +\sum_{\mathbf{t} m} C_{m}^{*}(\mathbf{t}) e^{i \mathbf{k t}}\left\langle R_{2 p}\left(r^{\prime}\right) Y_{1 m}\left(\mathbf{r}^{\prime}\right)\left|\left(\mathbf{e} \cdot \mathbf{r}^{\prime}\right)\right| e^{i \mathbf{k} \mathbf{r}^{\prime}}\right\rangle .
\end{aligned}
$$

For convenience, one introduces two vectors with cyclic components

$$
G_{m}(\mathbf{k}, \mathbf{e})=\sum_{\mathbf{t}} C_{m}^{*}(\mathbf{t})(\mathbf{e} \cdot \mathbf{t}) e^{i \mathbf{k t}}, \quad Z_{m}(\mathbf{k})=\sum_{\mathbf{t}} C_{m}^{*}(\mathbf{t}) e^{i \mathbf{k} \mathbf{t}} .
$$

Then Exp.(18) could be rewritten in a more compact form 


$$
\begin{gathered}
M(\mathbf{k}, \mathbf{e})=\sum_{m} G_{m}(\mathbf{k}, \mathbf{e})\left\langle R_{2 p}(r) Y_{1 m}(\mathbf{r}) \mid e^{i \mathbf{k r}}\right\rangle+ \\
\sum_{m} Z_{m}(\mathbf{k})\left\langle R_{2 p}(r) Y_{1 m}(\mathbf{r})|(\mathbf{e} \cdot \mathbf{r})| e^{i \mathbf{k r}}\right\rangle .
\end{gathered}
$$

Making use the familiar expansion (15) for the plane wave we rewrite (20) as follows

$$
\begin{gathered}
M_{\gamma \mu}^{(O)}(\mathbf{k}, \mathbf{e})=2 \sqrt{3 \pi} i B(k)\left(\mathbf{G}_{\gamma \mu} \cdot \mathbf{k}\right) / k+ \\
\sqrt{\frac{4 \pi}{3}}\left\{A_{0}(k)\left(\mathbf{Z}_{\gamma \mu} \cdot \mathbf{e}\right)-A_{2}(k) \frac{3(\mathbf{e} \cdot \mathbf{k})\left(\mathbf{Z}_{\gamma \mu} \cdot \mathbf{k}\right)-k^{2}\left(\mathbf{Z}_{\gamma \mu} \cdot \mathbf{e}\right)}{k^{2}}\right\}
\end{gathered}
$$

where we add the $\gamma \mu$ indexes label for the molecular orbital under consideration. Rather simple analytical expressions for the radial integrals $A_{0,2}, B$ in (21) could be obtained if to make use the simplest Slater $O 2 p$ radial wave function

$$
R_{2 p}(r)=\frac{1}{2 \sqrt{6}} \frac{1}{\sqrt{a_{p}^{3}}} \frac{r}{a_{p}} \exp \left\{-\frac{r}{2 a_{p}}\right\}
$$

Then

$$
\begin{gathered}
B(k)=\left\langle R_{2 p}(r) \mid j_{1}(k r)\right\rangle=\frac{64 \sqrt{6} a_{p}^{2} \sqrt{a_{p}} k}{3\left(1+4 a_{p}^{2} k^{2}\right)^{3}}, \\
A_{0}(k)=\left\langle R_{2 p}(r)|r| j_{0}(k r)\right\rangle=\frac{64 \sqrt{6} a_{p}^{2} \sqrt{a_{p}}\left(1-4 a_{p}^{2} k^{2}\right)}{\left(1+4 a_{p}^{2} k^{2}\right)^{4}}, \\
A_{2}(k)=\left\langle R_{2 p}(r)|r| j_{2}(k \rho)\right\rangle=\frac{512 \sqrt{6} a_{p}^{4} \sqrt{a_{p}} k^{2}}{\left(1+4 a_{p}^{2} k^{2}\right)^{4}} .
\end{gathered}
$$

The photoelectron energy dependence of quantities $B(k), A_{0}(k)$ and $A_{2}(k)$ given the $O 2 p$ radial parameter $a_{p}=0.52$ $\AA$ is shown in Fig.4.

\subsection{Expression for $M_{b_{1 g}}(\mathbf{k}, \mathbf{e})$}

Below we consider in detail the matrix element which specifies the contribution of the electron-removal process from the $b_{1 g}$ orbital to form the Zhang-Rice singlet.

For $\gamma \mu=b_{1 g}$ at rather large photon energy $E_{p h}>20 \mathrm{eV}$, but small binding energy $E<1 \div 2 \mathrm{eV}$ )

$$
M_{b_{1 g}}^{(C u)}(\mathbf{k}, \mathbf{e})=2 i \sqrt{\frac{3 \pi}{5}}\left\{D_{1}(k)+\frac{7}{2} D_{3}(k)\right\}\left(e_{x} \kappa_{x}-e_{y} \kappa_{y}\right),
$$

where $\kappa_{\alpha}=k_{\alpha} / k, \alpha=x, y, z$. Making use the numerical values for the coefficients $C_{m}^{\left(b_{1 g}\right)}(\mathbf{t})$ from Table 1 one might obtain

$$
\begin{array}{r}
M_{b_{1 g}}^{(O)}(\mathbf{k}, \mathbf{e})=i \sqrt{\frac{\pi}{3}}\left\{-3 a B(k)\left[\kappa_{x} e_{x} \cos \left(\frac{a k_{x}}{2}\right)-\kappa_{y} e_{y} \cos \left(\frac{a k_{y}}{2}\right)\right]-\right. \\
2\left[A_{0}(k)+A_{2}(k)\right]\left[e_{x} \sin \left(\frac{a k_{x}}{2}\right)-e_{y} \sin \left(\frac{a k_{y}}{2}\right)\right]+ \\
\left.6 A_{2}(k)\left[e_{x} \kappa_{x}+e_{y} \kappa_{y}\right]\left[\kappa_{x} \sin \left(\frac{a k_{x}}{2}\right)-\kappa_{y} \sin \left(\frac{a k_{y}}{2}\right)\right]\right\} .
\end{array}
$$

It should be emphasized that the photocurrent intensity at the BZ center in the case of the even $b_{1 g}$ orbital as well as for any other even $\gamma$ orbital turns to zero.

The bonding one-electron molecular $b_{1 g}$ orbital can be written as follows

$$
\Psi_{b_{1 g}}(\mathbf{r})=d_{b_{1 g}}(\mathbf{r}) \sin \theta_{b_{1 g}}+p_{b_{1 g}}(\mathbf{r}) \cos \theta_{b_{1 g}}
$$


where $\theta_{b_{1 g}}$ is an angular covalent mixing parameter. Then the photocurrent intensity will be proportional to

$$
\begin{gathered}
\left|\left\langle\Psi_{b_{1 g}}(\mathbf{k})|(\mathbf{e} \cdot \mathbf{r})| e^{i \mathbf{k r}}\right\rangle\right|^{2}=\left|M_{b_{1 g}}^{(C u)}\right|^{2} \sin ^{2} \theta_{b_{1 g}}+ \\
\left|M_{b_{1 g}}^{(O)}\right|^{2} \cos ^{2} \theta_{b_{1 g}}+\sin \theta_{b_{1 g}} \cos \theta_{b_{1 g}}\left\{\left(M_{b_{1 g}}^{(C u)}\right)^{*} M_{b_{1 g}}^{(O)}+M_{b_{1 g}}^{(C u)}\left(M_{b_{1 g}}^{(O)}\right)^{*}\right\} .
\end{gathered}
$$

\subsection{Expression for $M_{e_{u}}(\mathbf{k}, \mathbf{e})$}

As it was mentioned above, the oxygen $e_{u}$ states one might subdivide to $\sigma$ and $\pi$ orbitals. Due to a strong $O 2 p-O 2 p$ coupling they hybridize to form bonding and antibonding molecular $e_{u}$ orbitals [29]. The bonding one-electron molecular $e_{u}$ orbital can be written as follows

$$
\Psi_{e_{u} \mu}(\mathbf{r})=p_{e_{u} \mu}^{(\pi)}(\mathbf{r}) \sin \theta_{e_{u}}+p_{e_{u} \mu}^{(\sigma)}(\mathbf{r}) \cos \theta_{e_{u}}, \quad \mu=x, y,
$$

where $\theta_{e_{u}}$ is a covalent mixing parameter. Then the photocurrent intensity will be proportional to

$$
\begin{aligned}
& \sum_{\mu}\left|\left\langle\Psi_{e_{u} \mu}(\mathbf{r})|(\mathbf{e} \cdot \mathbf{r})| e^{i \mathbf{k r}}\right\rangle\right|^{2}=\sum_{\mu}\left|M_{\mu}^{(\pi)}\right|^{2} \sin ^{2} \theta_{e_{u}}+ \\
& \sum_{\mu}\left|M_{\mu}^{(\sigma)}\right|^{2} \cos ^{2} \theta_{e_{u}}+\sin 2 \theta_{e_{u}} \sum_{\mu} M_{\mu}^{(\pi)} M_{\mu}^{(\sigma)} .
\end{aligned}
$$

Making use of the coefficients from Table 1 one might obtain general expressions for matrix elements in the case of $\sigma$ states $\gamma \mu=e_{u} x$ or $\gamma \mu=e_{u} y$ :

$$
\begin{gathered}
M_{\mu}^{(\sigma)}(\mathbf{k}, \mathbf{e})=-\sqrt{6 \pi} a B(k) e_{\mu} \kappa_{\mu} \sin \left(\frac{a k_{\mu}}{2}\right)+ \\
\sqrt{\frac{8 \pi}{3}}\left\{\left(A_{0}(k)+A_{2}(k)\right) e_{\mu}-3 A_{2}(k) \kappa_{\mu}(\mathbf{e} \cdot \kappa)\right\} \cos \left(\frac{a k_{\mu}}{2}\right) \\
(\mu=x, y) .
\end{gathered}
$$

Similarly, one might obtain general expressions for matrix elements in the case of $\pi$ states $\gamma \mu=e_{u} x$ and $\gamma \mu=e_{u} y$ :

$$
\begin{gathered}
M_{x}^{(\pi)}(\mathbf{k}, \mathbf{e})=-\sqrt{6 \pi} a B(k) e_{y} \kappa_{x} \sin \left(\frac{a k_{y}}{2}\right)+ \\
\sqrt{\frac{8 \pi}{3}}\left\{\left(A_{0}(k)+A_{2}(k)\right) e_{x}-3 A_{2}(k) \kappa_{x}(\mathbf{e} \cdot \kappa)\right\} \cos \left(\frac{a k_{y}}{2}\right), \\
M_{y}^{(\pi)}(\mathbf{k}, \mathbf{e})=-\sqrt{6 \pi} a B(k) e_{x} \kappa_{y} \sin \left(\frac{a k_{x}}{2}\right)+ \\
\sqrt{\frac{8 \pi}{3}}\left\{\left(A_{0}(k)+A_{2}(k)\right) e_{y}-3 A_{2}(k) \kappa_{y}(\mathbf{e} \cdot \kappa)\right\} \cos \left(\frac{a k_{x}}{2}\right) .
\end{gathered}
$$

\section{Modelling the polaronic matrix element effects in ARPES spectroscopy}

Orbital $b_{1 g}-e_{u}$ quasidegeneracy and polaronic nature of the photohole results in a complicated structure of the energy and momentum dependence of the photointensity. Below, we would like to present some examples of the straightforward model calculations of the matrix elements effects in ARPES. First of all we should address to the k-dependence of the single $\mathrm{CuO}_{4}$ center contribution, the polaron form-factor effects, the photon polarization effects, and interference effects, caused by $b_{1 g}-e_{u}$ quasidegeneracy.

\subsection{Matrix element effects for isolated $\mathrm{CuO}_{4}$ center}

The Figure 5 shows the contour-plots (the darker the color, the bigger the photointensity) for the quantities $\left|M_{\gamma \mu}(\mathbf{k})\right|^{2}$ which describe a partial one-center form-factor contribution to the photo-current intensity. The kdependence of the polarization averaged $\overline{\mid\left\langle\left.\Psi_{b_{1 g}}(\mathbf{r})|(\mathbf{e} \cdot \mathbf{r})| e^{i \mathbf{k r}\rangle}\right|^{2}\right.}$ for $\theta_{b_{1 g}}=-0.33 \pi$ is shown in Fig.5a. Simply speaking, this is a contribution of the conventional model Zhang-Rice singlet. From left to right here we present 
the purely $\mathrm{Cu} 3 d$, purely $\mathrm{O} 2 p$, and the total contributions, respectively. One should note the complex k-dependence of the oxygen contribution as compared with the copper one. The $\mathbf{k}$-dependence of the polarization and orbitally averaged $\overline{\sum_{\mu}\left|\left\langle\Psi_{e_{u} \mu}(\mathbf{r})|(\mathbf{e} \cdot \mathbf{r})| e^{i \mathbf{k r}}\right\rangle\right|^{2}}$ contribution of the purely oxygen $e_{u}$ electron-removal state for $\theta_{e_{u}}=0.30 \pi$ is shown in Fig.5b. From left to right there are presented the $\pi, \sigma$, and hybrid (interference) $\pi-\sigma$ contributions, respectively. It should be noted that the $\pi$ and $\sigma$ partial contributions have a rather different $\mathbf{k}$-dependence.

To illustrate the photon polarization effects we present several examples of the angular $(\vec{k})$ dependence of the photointensity for the "parallel" $(\vec{e} \| \vec{k})$, and "perpendicular" $(\vec{e} \perp \vec{k})$ polarizations, respectively, calculated with the help of Exps. (23)-(24). The Figure 6a relates to the Cu3d partial contribution to the photointensity with creation of photo-hole in ZR-singlet state for the parallel $\left(\propto \cos ^{2} 2 \phi\right)$, or perpendicular $\left(\propto \sin ^{2} 2 \phi\right)$ polarization, respectively. The Figure $6 \mathrm{~b}$ relates to the corresponding $O 2 p$ partial contribution. Here, one should note the more complex form of the angular dependence due to the essentially different structure of the respective matrix elements. However, the $k$ modulus dependence of the polar plots in Fig.6b is rather weak. So, in a whole, the polarization effects in both cases appear to be qualitatively, and even quantitatively similar. A comparative analysis of the Figures 5 and 6 indicates strong impact of the photon polarization effect on the final momentum dependence of the photocurrent intensity.

\subsection{Matrix element effects for isolated large polaron}

To illustrate an important role of the matrix element effects, we consider below a four-, and five-center model of the immobile large PJT polaron generated by the isolated valent ${ }^{1} A_{1 g}-{ }^{1,3} E_{u}$ manifold of the $C u O_{4}^{5-}$ center, that is assuming a localization of the photohole either in $b_{1 g}$ or $e_{u}$ orbital on the $\mathrm{CuO}_{4}$ center.

The Figure 7a,b shows the contour-plots for a number of quantities $\left|f_{\Gamma M}^{(\gamma \mu)}(\mathbf{k})\right|^{2}$ which represent a peculiar " $k$ portrait" of the hole density within $N$-center large polaron $(N=4$, Fig.7a, $N=5$, Fig.7b), and describe a partial polaronic form-factor contribution to the photo-current intensity. Again we see complex and various momentum dependencies, reflecting both the hole symmetry and its distribution in large polaron.

The Figure 8 shows the contour-plots for a number of quantities $\left|\mathcal{M}_{\Gamma M}(\mathbf{k})\right|^{2}\left(\Gamma=B_{1 g}, E_{u}\right)$ which describe a polarization and orbital averaged overall matrix element effect in partial $\Gamma$ contribution to the photo-current intensity with one-center hole basis consisting of the $b_{1 g^{-}}$, and $e_{u}$-orbitals. The top figures present the total contribution, while below there are shown the partial $b_{1 g^{-}}, e_{u^{-}}$, and interference $b_{1 g}-e_{u^{-}}$-contributions, respectively. Interestingly, the interference term looks similarly in both cases. One should notice the nonzero contribution of the $e_{u}$ states to the photointensity in the BZ center ( $\Gamma$-point) for the $E_{u}$ type polaron.

To illustrate the photon polarization effects we have calculated the angular $(\vec{k})$ dependence of the partial photointensity related to photo-hole creation in $\Psi_{B_{1 g}}^{e_{u}}$ state for the "parallel", and "perpendicular" polarizations, respectively (Fig.9). Interestingly, the dependence is qualitatively similar to the case of the ZR-singlet, at least for the high-symmetry directions. In other words, the polarization dependence alone could not distinguish the ZRsinglet among other terms with the relevant symmetry. The Figures 8 and 9 convincingly illustrate the role played by the matrix element effects, including the form-factor, orbital symmetry, photon polarization, and interference effects. As we see, for a quantitative comparison with experiment, it is necessary to add the ARPES amplitudes instead of intensities, taking into account the polarization and energy of the incident photons, and the direction of the photoemitted electrons.

So, one might unambiguously say that the matrix element effects result in a complex $\mathbf{k}$ dependence of the photocurrent intensity which has to be taken into account when addressing such issues as Fermi surface. Indeed, the above model illustrations provide a wide choice of the "Fermi surface"-like behavior. Neglecting the matrix element effects results in erroneous conclusions concerning the electronic structure of the electron-removal states.

\section{Conclusions}

We had not for an object the detailed fitting of the experimental photoemission spectra, as we consider this problem in the meantime to be very complicated. At present, there is no generally accepted model for the large PJT polaron in copper oxides and appropriate ARPES spectral functions $A(\omega)$, and it leads to uncertainties in quantitative interpretation of the experimental data.

Nevertheless, we see that the low-energy ARPES spectra could be originated from the hole polaronic excitations which complex spectral shape is strongly affected by the soft lattice and spin fluctuations. These usually have to result in a complex ARPES spectral shape with a rather narrow purely electronic (coherent) peak and structureless (incoherent) background which describes the excitations accompanied by the emission and absorption of bosons (phonons, spin waves ). One should notice that the polaronic spectral response can spread over a wide energy range of about several tenths of eV. Experimental spectral shape of the intensity is, qualitatively, compatible with that expected for spectral response of the PJT polaron [26]. Perhaps, namely the polaron-like entity formation could explain the extremely narrow and intense peak lying below the Fermi energy and being the most intriguing feature of ARPES in all the high- $T_{c}$ cuprates. We also could propose that many unusual features of the cuprate ARPES including the $\vec{k}$-dependence of spectral shape can be understood and described without any Fermi-surface, large or small. 
A qualitative comparison of experimental ARPES spectra and model calculations of the matrix element effects allows to make a number of important conclusions:

1. A model of the dispersionless valent ${ }^{1} A_{1 g}-{ }^{1,3} E_{u}$ multiplet of the two-hole $C u O_{4}^{5-}$ center and large $(N=4$, or 5) non-adiabatic PJT polaron is quite enough compatible with experimental ARPES data. Moreover, this is capable to describe some rather subtle spectral ARPES features, including the "remnant Fermi surface" effect without any reference to itinerant band-like states. It should be emphasized one more the principal role of ${ }^{1,3} E_{u}$ term determining the non-zero ARPES response in $\Gamma$ point.

2. The k-dependent spectral weight transfer for the large PJT polaron could be a natural origin of a "seeming" hole dispersion when to be described in a single-band model. At the same time, the polaronic transport described by the Hubbard-like model also could provide the natural explanation of the dispersion observed. Apparently, the straightforeward assignement of the photocurrent intensity maxima to that of the quasiparticle spectral density may result in erroneous conclusions.

3. Interpretation of the photoemission spectra for strongly correlated oxides needs a caution and careful account for multi-band effects and matrix element effects, especially in what concerns the $\mathbf{k}$-dependence of the spectral weight, and Fermi surface assignement. Moreover, the polaronic nature of the photo-hole gives rise to the problem of the "third dimension" of polaron, or to its correlation length in $c$-direction resulting in a number of important consequences concerning the photon energy dependence of photointensity.

All these conclusions cast doubt on results of numerous papers with simplified interpretation of the low-energy ARPES data for $\mathrm{Sr}_{2} \mathrm{CuO}_{2} \mathrm{Cl}_{2}$ made in the framework of the various single-band versions of the $t-J$ model [9.10], and aimed the interpretation of the "experimental quasiparticle dispersion law" supposedly determined from positions of the photocurrent intensity maxima. Unfortunately, the available experimental data do not allow to make so far the reliable conclusions on the nature of the low-energy ARPES feature. The ARPES data have to be considered with great care since it is most probable that they do not reflect straightforwardly the bulk density of states (DOS) of the quasiparticle excitations. In our opinion, a similar situation with the ARPES data interpretation occurs for many other strongly correlated oxides, including the superconducting cuprates, that forces to consider with caution many principal conclusions which are founded on the ARPES data.

Concluding, one should be noted that the elaboration of adequate theory of the electronic spectra for the strongly correlated oxides needs at present not only solution of a number of complex theoretical problems, but the more perfect experimental data with complete spectral, angular, and polarization analysis.

The research described in this publication was made possible in part by Award No.REC-005 of the U.S. Civilian Research \& Development Foundation for the Independent States of the Former Soviet Union (CRDF). The authors acknowledge a partial support from the Russian Ministry of Education, grant \# 97-0-7.3-130. 


\section{Figure captions}

Fig.1. Schematic representation of the electron (hole) density distribution in the hybrid $C u 3 d O 2 p b_{1 g^{-}}$, and purely oxygen $e_{u}(\sigma, \pi)$ molecular orbitals, assumed to be main components of the first electron-removal state in insulating copper oxides.

Fig.2. Schematic structure of the large polaron: (a) $N=4$, (b) $N=5$.

Fig.3. Dependence of the $C u 3 d$-atomic radial parameters $D_{1}(E)$ and $D_{3}(E)$ on the photoelectron energy $\left(a_{d}=\right.$ $0.35 \AA)$.

Fig.4. Dependence of the $O 2 p$-atomic radial parameters $B(E), A_{0}(E)$ and $A_{2}(E)$ on the photoelectron energy $\left(a_{p}=0.52 \AA\right)$.

Fig.5. Contour-plots for the $\vec{k}$-dependence of the partial one-center form-factor contribution to the photo-current intensity for the depolarized photons: a) $\gamma \mu=b_{1 g}$, from left to right the $C u 3 d, O 2 p$, and the hybrid $C u 3 d O 2 p$ $\left(\theta_{b_{1 g}}=-0.3 \pi\right)$ contributions, respectively; b) $\gamma \mu=e_{u}$, from left to right the $\pi, \sigma$ and hybrid $\sigma \pi\left(\theta_{e_{u}}=0.4 \pi\right)$ contributions, respectively.

Fig.6. Photon polarization effects. Angular $(\vec{k})$ dependence of the ZR-singlet partial contribution to photointensity for the "parallel" $(\vec{e} \| \vec{k})$, and "perpendicular" $(\vec{e} \perp \vec{k})$ polarizations, respectively: a) Cu3d partial contribution; b) $O 2 p$ partial contribution. Numbers near curves indicate the $k$ values.

Fig.7. Contour-plots for polaronic formfactors $\left|f_{\Gamma M}^{(\gamma \mu)}(\mathbf{k})\right|^{2}$ which describe a partial polaronic form-factor contribution to the photo-current intensity, and represent a peculiar " $k$-portrait" of the hole density within $N$-center large polaron: a) $N=4$, b) $N=5$.

Fig.8. Contour-plots for quantities $\left|\mathcal{M}_{\Gamma M}(\mathbf{k})\right|^{2}\left(\Gamma=B_{1 g}, E_{u}\right)$ which describe a polarization and orbital averaged overall matrix element effect in partial $\Gamma$ contribution to the photo-current intensity. The top figures present the total contribution, while below there are shown the partial $b_{1 g^{-}}, e_{u^{-}}$, and interference $b_{1 g}-e_{u^{-}}$-contributions, respectively.

Fig.9. Photon polarization effects. Angular $(\vec{k})$ dependence of the partial contribution to photointensity related to photo-hole creation in $\Psi_{B_{1}}^{e_{u}}$ state for the "parallel", and "perpendicular" polarizations, respectively. Different curves correspond the same $k$ values as in Fig.6b.

[1] V.I. Anisimov, F. Aryasetiawan, A. Lichtenstein, J. Phys.: Condens. Matter, 9, 767 (1997).

[2] A.S. Alexandrov and C.J. Dent, cond-mat/9905185 13 May 1999.

[3] J.M. Eroles, C. D. Batista and A.A. Aligia, cond-mat/9812325 18 Dec 1998.

[4] A. Bansil and M. Lindroos, cond-mat/9910496 29 Okt 1999.

[5] S. Haffner, C.G. Olson, L.L. Miller, D.W. Lynch, Phys. Rev. B61, 2000, p. 14378.

[6] S. Haffner, D.M. Brammeier, C.G. Olson, L.L. Miller, D.W. Lynch, arXiv:cond-mat/0006366 23 Jun 2000.

[7] E. Seibel and H. Winter. J. Phys.: Condens. Matter 10 (1998) 5197-5216.

[8] V. A. Gavrilov, S. G. Ovchinnikov. Fiz. Tverd. Tela, B40, 2, 184-190, 1998.

[9] B.O. Wells, Z.-X. Shen, A. Matsuura, D. M. King, M.A. Kastner, M. Greven and R.J. Birgenau, Phys. Rev. Lett., 74, 964, (1995).

[10] A. Nazarenko, K. J. E. Vos, S. Haas et al. J. Supercond. 8, 671 (1995).

[11] A. Zibold, H.L. Liu, S.W. Moore, J.M. Graybeal, D.B. Tanner, Phys. Rev. B53, 1996, p. 11734.

[12] T. Boeske, O. Knauff, R. Neudert, M. Kielwein, M. Knupfer, M.S. Golden, J. Fink, H. Eisaki, S. Ushida, K. Okada, A. Kotani, Phys. Rev. B56, 1997, p. 3438.

[13] A. Fujimori, Y. Tokura, H. Eisaki, H. Takagi, S. Uchida and M. Sato, Phys. Rev. Lett., 40, 10, 7303, (1989).

[14] S. Haffner, R. Neudert, M. Kielwein, M. Knupfer, M.S. Golden, K. Ruck, G. Krabbes, J. Fink, H. Rosner, R. Hayn, H. Eisaki, S. Ushida, Z. Hu, M. Domke, G. Kaindl, Phys. Rev. B57,1997, p. 3672.

[15] T. Boeske, K. Maiti, O. Knauff, K. Ruck, M.S. Golden, G. Krabbes, J. Fink, T. Osafune, N. Motoyama, H. Eisaki, S. Ushida, Phys. Rev. B57,1997, p. 138.

[16] Y.Y. Wang, F.C. Zhang, V.P. Dravid, K.K. Ng, M.V. Klein, S.E. Schnatterly, and L.L. Miller, Phys. Rev. Lett., 77, 1996, p. 1809.

[17] R. Neudert, T. Boeske,O. Knauff, M. Knupfer, M.S. Golden, G. Krabbes, J. Fink, H. Eisaki, S. Ushida, Physica B 230-232, 1997, p. 847.

[18] J. Fink, R. Neudert, H.C. Schmelz, T. Boeske, O. Knauff, S. Haffner, M. Knupfer, M.S. Golden, G. Krabbes, H. Eisaki, S. Uchida, Physica B 237-238, 1997, p. 93.

[19] R. Hayn, H. Rosner, V. Yu. Yushankhai, S. Haffner, C. Duerr, M. Knupfer, G. Krabbes, M. S. Golden, J. Fink, H. Eshrig, D. J. Singh, N.T. Hien, A.A. Menovsky, Ch. Jung and G. Reichardt., Phys. Rev., B60,1, 645(1999).

[20] F. Ronning, C. Kim, D.L. Feng et al., condmat// 9903151; Science, 282, 2067 (1998).

[21] S. LaRosa, I. Vobornik, F. Zwick et.al., Phys. Rev. B56,1997, R525.

[22] C. Kim, P.J. White, Z.-X. Shen et.al., Phys. Rev. Lett., 80, 1998, 4245.

[23] C. Dürr, S. Legner, R. Hayn et al., arXiv:cond-mat/0007283 17 Jul 2000.

[24] J.E. Hirsch and S. Tang. Phys.Rev.B, 40, 4,p.2179 (1989); J.E. Hirsch, in "Polarons and bipolarons in high- $T_{c}$ super- 
conductors and related materials", eds E.K.H. Salje, A.S. Alexandrov and W.Y. Liang, Cambridge University Press, 1995, p. 234..

[25] Gennadi I. Bersuker, John B. Goodenough, Physica C 274 (1997) 267-285.

[26] I. B. Bersuker and V. Z. Polinger, Vibronic Interactions in Molecules and Crystals, Springer-Verlag, Berlin 1989.

[27] Gerald F. Dionne, J. Appl. Phys. 69 (1991) 5194.

[28] A.S. Moskvin, Yu.D. Panov, E.N. Kondrashov, R.A. Istomin (unpublished)

[29] A.S. Moskvin, N.N. Loshkareva, Yu.P. Sukhorukov, M.A. Sidorov, A.A. Samokhvalov, JETP 105 (1994) 967.

[30] J. Tanaka, K. Kamiya and C. Tanaka, Physica C 161, 451 (1989).

[31] J. Tanaka and C. Tanaka, J. Phys. Chem. Solids 59, 1861 (1998).

[32] J.B. Goodenough, J.-S. Zhou, Phys. Rev. 49, 4251 (1994).

[33] E. Dagotto, Rev.Mod. Phys., 66, 763 (1994).

[34] A.S.Moskvin, Yu.D.Panov, JETP 84 (1997) 354; Physica Status Solidi (b), 212 (9),141, 1999; Journ. Phys. Chem. of Solids, 60, 607, 1999.

[35] A.S.Moskvin, A.S.Ovchinnikov, JMMM, 186, 288, 1998; Physica C , 296, 250, 1998.

[36] S. Yoshinari, P.C. Hammel, Z. Fisk, Phys. Rev. Lett. 77 (1996) 2016.

[37] Yoshinari Y., Physica C 276, 147 (1997).

[38] L.H. Tjeng, B. Sinkovic, N.B. Brookes et al., Phys. Rev. Lett. 78 (1997) 1126.

[39] P.W. Leung, B.O. Wells and R.J. Gooding, cond-mat/9702016 3 Feb 1997.

[40] D. A. Varshalovich, A. N. Moskalev, V. K. Khersonskii. Quantum Theory of Angular Momentum (World Scientific, Singapore, 1988). 
TABLE I. The coefficients $C_{m}^{(\gamma \mu)}(\mathbf{t})$.

\begin{tabular}{||c|c|c|c|c||}
\hline \hline$C_{m}^{(\gamma \mu)}(\mathbf{t})$ & $\frac{\mathbf{x}}{2}$ & $-\frac{\mathrm{x}}{2}$ & $\frac{\mathrm{y}}{2}$ & $-\frac{\mathrm{y}}{2}$ \\
\hline$C_{+1}^{\left(b_{1} g\right)}(\mathbf{t})$ & $\frac{1}{2 \sqrt{2}}$ & $-\frac{1}{2 \sqrt{2}}$ & $\frac{i}{2 \sqrt{2}}$ & $-\frac{i}{2 \sqrt{2}}$ \\
\hline$C_{-1}^{\left(b_{11} g\right.}(\mathbf{t})$ & $-\frac{1}{2 \sqrt{2}}$ & $\frac{1}{2 \sqrt{2}}$ & $\frac{i}{2 \sqrt{2}}$ & $-\frac{i}{2 \sqrt{2}}$ \\
\hline$C_{+1}^{(\sigma x)}(\mathbf{t})$ & $-\frac{1}{2}$ & $-\frac{1}{2}$ & 0 & 0 \\
\hline$C_{-1}^{(\sigma x)}(\mathbf{t})$ & $\frac{1}{2}$ & $\frac{1}{2}$ & 0 & 0 \\
\hline$C_{+1}^{(\sigma y)}(\mathbf{t})$ & 0 & 0 & $\frac{i}{2}$ & $\frac{i}{2}$ \\
\hline$C_{-1}^{(\sigma y)}(\mathbf{t})$ & 0 & 0 & $\frac{i}{2}$ \\
\hline$C_{+1}^{(\pi x)}(\mathbf{t})$ & 0 & 0 & $-\frac{1}{2}$ & $-\frac{1}{2}$ \\
\hline$C_{-1}^{(\pi x)}(\mathbf{t})$ & 0 & 0 & $\frac{1}{2}$ \\
\hline$C_{+1}^{(\pi y)}(\mathbf{t})$ & $\frac{i}{2}$ & $\frac{i}{2}$ & 0 & 0 \\
\hline$C_{-1}^{(\pi y)}(\mathbf{t})$ & $\frac{i}{2}$ & $\frac{i}{2}$ & 0 & 0 \\
\hline \hline
\end{tabular}

